\title{
A step in the right direction
}

\author{
Vincent T DeVita, Jr
}

One of the main roadblocks to drug development is the testing of a new agent in heavily pretreated patients with any type of cancer. By the time the highly resistant cancer cell 'sees' the new drug, its genome has been highly destabilized and, by dint of many mutations, translocations and exposure to several chemical entities, the cell has selected pathways for growth and avoidance of apoptosis that are highly advantageous to its continued expansion. It is surprising that such tumor cells respond to drugs at all. We are more likely to be misled than informed by information from studies done under these circumstances. Yet such studies are the 'gold standard', as the FDA states it, especially when a drug is tested in a randomized trial that compares the new drug, either alone or in combination with another agent, against the standard treatment, with survival as the endpoint.

The FDA is, therefore, to be congratulated for approving, in July of this year, the use of the drug gemcitabine $\left(\right.$ Gemzar $^{\circledR}$, Eli Lilly and Company, Indianapolis, IN) in combination with carboplatin for the treatment of patients with advanced, pretreated, ovarian cancer, ignoring the advice of the Oncologic Drug Advisory Committee, who thought approval should be withheld in the absence of a survival advantage associated with the use of gemcitabine. The study of 356 European patients showed an improvement in progression-free survival of only 2.8 months associated with the use of gemcitabine (Pfisterer $\mathrm{J}$ et al. [2006] $\mathrm{J}$ Clin Oncol 24: 4699-4707). This author is surprised at the recommendation of the Oncologic Drug Advisory Committee, whose members should know that approval under these circumstances opens pathways for more useful integration into earlier stage disease.
Hopefully the FDA will next

re-examine

the use of

response

rates for the

approval of

new drugs, but

approval of

gemcitabine...

is a step in the

right direction.

VT DeVita, Jr is Editor-in-Chief of Nature Clinical Practice Oncology.

Competing interests The author has declared associations with ImClone. See the article online for full details of the relationship.

www.nature.com/clinicalpractice doi:10.1038/ncponc0669
Gemcitabine is not a new drug in the traditional sense, and FDA approval follows on the heels of recognition by practitioners that it might be beneficial in the treatment of ovarian cancer. In addition, gemcitabine is not that expensive, relatively speaking. If it was an expensive new agent we could have expected negative press about spending thousands of dollars for a drug that only prolongs life by a miserly 2.8 months. This is a common theme in the lay press and ignores the next major benefit of approving new therapies that have marginal effects in advanced disease, that is, the likely usefulness of the same treatments applied in the 'the adjuvant setting' for patients who present with localized disease. For example, I often hear that despite the use of complicated expensive combinations of drugs to treat patients with metastatic colorectal cancer, we don't cure any patients and only prolong survival by a marginal amount. Yet, the reason there has been a more than $40 \%$ decline in national mortality from colorectal cancer in the US is largely because of the application of these treatments in earlystage disease. The same can be said for breast cancer treatment, where the $90 \%$ of patients who present with localized disease benefit from programs tested in the $10 \%$ who present with metastatic disease. The validation of the Norton-Simon hypothesis, previously reviewed in this journal (Simon R and Norton L [2006] Nat Clin Pract Oncol 3: 406-407), has taught us the perils associated with using survival as an endpoint and ignoring response rate, especially the complete response rate, as a surrogate endpoint for the effectiveness of a new drug. Hopefully the FDA will next re-examine the use of response rates for the approval of new drugs, but approval of gemcitabine for ovarian cancer is a step in the right direction. 